\title{
ACHADO INCIDENTAL DE AGENESIA DO LOBO HEPÁTICO ESQUERDO EM PACIENTE COM COLECISTITE AGUDA
}

\author{
Incidental finding of agenesis of the left hepatic lobe in a patient with acute chole- \\ cystitis
}

\author{
Bruno da Costa MARTINS, Marcus Fernando Kodama Pertille RAMOS, \\ Roberto de CLEVA, Bruno ZILBERSTEIN
}

ABCDDV/626

Martins BC, Ramos MFKP, Cleva R, Zilberstein B. Achado incidental de agenesia do lobo hepático esquerdo em paciente com colecistite aguda. ABCD Arq Bras Cir Dig 2008;21(4): 208-10

RESUMO - Introdução - Anomalias da morfologia hepática são entidades raras causadas por desenvolvimento embriológico anormal do fígado. Podem ser classificadas como anomalias devido a desenvolvimento defeituoso do fígado (agenesia, aplasia e hipoplasia) ou devido ao desenvolvimento excessivo (lobos acessórios). Relato do caso - Paciente de 48 anos, do sexo feminino, obesa (IMC=40), que procurou o serviço de emergência com quadro de colecistite aguda. A paciente foi submetida a colecistectomia videolaparoscópica e durante o procedimento cirúrgico foi verificada a agenesia do lobo hepático esquerdo. A vesícula biliar estava em sua topografia habitual e não havia parênquima hepático à esquerda do ligamento falciforme. A colecistectomia foi realizada sem intercorrências e uma banda gástrica foi colocada para o tratamento da obesidade mórbida. Ela recebeu alta após 60 horas

DESCRITORES - Colecistectomia. Anormalidades do sistema digestório.

\section{INTRODUÇÃO}

As anomalias da morfologia hepática são entidades distintas das variações anatômicas da morfologia hepática. Variações anatômicas são frequentes e ocorrem durante o desenvolvimento normal do órgão. Elas correspondem a variações da distribuição dos territórios hepáticos ${ }^{2}$. De modo contrário, as anomalias da morfologia hepática são raras, e correspondem a defeitos do desenvolvimento do órgão ${ }^{16}$. De acordo com Pages et al. ${ }^{16}$, as anomalias da morfologia relacionadas com defeitos do desenvolvimento podem ser divididas em agenesia (ausência de um lobo que é substituído por tecido fibroso); aplasia (um dos lobos é pequeno e sua estrutura é anormal, com poucas trabéculas hepáticas, numerosos ductos biliares e vasos sanguíneos anormais); e hipoplasia (um dos lobos é pequeno, mas possui estrutura normal). É importante enfatizar, que para o cirurgião é mais importante diferenciar anomalias congênitas do desenvolvimento hepático da atrofia hepática decorrente de uma doença biliar ou vascular adquirida. As anomalias de morfologia relacionadas com excesso de desenvolvimento correspondem aos lobos acessórios, tanto sésseis quanto pediculados.

$\mathrm{O}$ achado operatório de uma agenesia total do lobo hepático esquerdo em um paciente com colecistite aguda motivou a presente investigação e revisão da literatura.

Trabalho realizado no Serviço de Cirurgia de Estômago do Hospital das Clínicas da Faculdade de Medicina da Universidade de São Paulo, São Paulo, SP, Brasil.

Correspondência: Bruno da Costa Martins, e-mail: bcm.bruno@gmail.com

\section{RELATO DO CASO}

Paciente de 48 anos do sexo feminino, com obesidade mórbida ( $\mathrm{IMC}=40$ ), foi admitida em pronto socorro com história clínica típica de colecistite aguda. Ultrassonografia abdominal demonstrou distensão da vesícula biliar com espessamento da parede e presença de microlitíase, sem nenhuma evidência de qualquer alteração da morfologia hepática.

A paciente foi submetida a um procedimento de videolaparoscopia durante o qual foi evidenciado a agenesia do lobo hepático esquerdo, com a ausência de tecido hepático à esquerda do ligamento falciforme. $\mathrm{O}$ lobo hepático direito e o segmento I estavam presentes sem qualquer sinal macroscópico de anomalias. A vesícula biliar estava situada em sua topografia habitual, e a anomalia hepática não interferiu na técnica cirúrgica empregada, sendo realizada a colecistectomia sem intercorrências. Devido à obesidade mórbida, uma banda gástrica ajustável foi colocada ao redor do estômago, com consentimento prévio da paciente. A evolução pósoperatória ocorreu sem intercorrências, e a paciente recebeu alta hospitalar após 60 horas sem queixas.

\section{DISCUSSÃO}

A agenesia do lobo hepático esquerdo é condição rara, tendo sido relatada a primeira vez por Rolleston ${ }^{18} \mathrm{em} 1893$ e posteriormente por Messing ${ }^{14}$ em 1932. É difícil estabelecer sua prevalência, ao revisar 19.000 necrópsias Merril ${ }^{13}$ encontrou apenas um caso.

A maioria dos pacientes são usualmente assintomáticos, no entanto, esta anomalia pode afetar a anatomia das estru- 
turas vizinhas, causando diferentes sintomas. O órgão mais comumente afetado é o estômago, que tende a ocupar o espaço sub-diafragmático onde normalmente está localizado o lobo hepático esquerdo ${ }^{16}$. $\mathrm{O}$ eixo do estômago adquire posição horizontal que pode causar um volvo gástrico permanente ou transitório ${ }^{1,12}$. No presente caso, o estômago estava localizado em sua posição habitual sem qualquer desvio do seu eixo, o que possibilitou a colocação de uma banda gástrica com facilidade devido à falta de necessidade de afastar o lobo hepático esquerdo.

Outras anomalias associadas também podem ocorrer como colelitíase, vesícula biliar flutuante, doença ulcerosa péptica, hérnia de hiato e malformações diafragmáticas ${ }^{1,11}$. Esta paciente apresentava colelitíase com colecistite aguda. Provavelmente esta situação ocorreu devido à obesidade mórbida da paciente, visto que 12 a $30 \%$ dos obesos mórbidos têm colelitíase ${ }^{4}$. Além do estômago, outros órgãos também podem apresentar alteração do seu posicionamento anatômico. O lobo hepático direito pode apresentar tamanho normal ou tornar-se aumentado deixando seu eixo verticalmente orientado ${ }^{2,18}$. Quando esta situação ocorre, o cólon ascendente é tracionado medialmente. Alterações de posicionamento da vesícula biliar também já foram descritas como a verticalização, posicionamento medial em relação ao pedículo hepático e vesícula flutuante ${ }^{11}$.

Anomalias do desenvolvimento do lobo hepático direito foram relatadas pela primeira vez por $\mathrm{Heller}^{6} \mathrm{em} 1870$. Nesses casos o suprimento vascular hepático usualmente é realizado através de uma rede muito fina, embora em alguns casos uma veia setorial normal possa existir. A vesícula está frequentemente posicionada à direita do fígado, contra o diafragma em uma posição vertical ${ }^{2}$. Malformações da cúpula diafragmática direita, pulmonares e alteração da rotação intestinal também podem ocorrer ${ }^{9}$. A agenesia ou hipoplasia do lobo hepático direito pode predispor ao desenvolvimento de hipertensão portal e varizes de esôfago, especialmente quando o lobo esquerdo não está aumentado ${ }^{2,17}$. Nesses casos, os pacientes podem apresentar episódios de sangramento das varizes esofágicas antes dos 30 anos de idade. A provável etiologia da hipertensão portal parece ser devido à diminuição dos ramos intra-hepáticos da veia porta que não foi compensada pelo aumento do lobo esquerdo 7 . Entretanto, existem relatos de pacientes com lobo hepático esquerdo não aumentado e sem evidências de hipertensão portal. Desse modo, a verdadeira causa da hipertensão portal nos pacientes com agenesia ou hipogenesia do lobo direito ainda precisa ser elucidada ${ }^{2}$.

O diagnóstico pré-operatório da ausência de um lobo hepático pode ser feito pela ultrasonografia abdominal ou pela tomografia computadorizada. A ausência de parênquima hepático à esquerda da fossa da vesícula biliar é considerada diagnóstica por alguns autores ${ }^{15}$. O lobo hepático direito pode apresentar tamanho normal ou estar aumentado, assim como o lobo caudado. Elevação do antro gástrico, estômago em formato de "U" e posicionamento alto do bulbo duodenal também já foram relatados ${ }^{1,2,8,15}$.

Os fatores envolvidos na etiologia das anomalias do desenvolvimento hepático ainda precisam ser esclarecidos. Alguns autores sugerem que essas anomalias decorrem de fluxo portal inadequado durante a embriogênese $e^{2,5,7,9}$. Entretanto também é possível que o fator desencadeante das anomalias ocorra antes da organização do sistema hepático venoso portal definitivo². Relatos recentes se focaram na descrição anatômica de três casos de hipoplasia do lobo hepático esquerdo ${ }^{3,10}$. Em todos fusão peritoneal espessa foi observada ao redor do fígado. Durante a dissecção, a veia hepática esquerda estava presente com ramos terminais similares. A veia nunca transformou-se em um remanescente fibroso. Cho et al. ${ }^{3}$, especularam que a formação de uma cicatriz ao redor da porta hepatis na fase tardia do desenvolvimento embrionário, ou mesmo durante o crescimento pós-natal, envolvendo o ligamento redondo e a veia porta esquerda causaria hipoplasia secundária. Lee et $\mathrm{al}^{10}$, consideram que constrição membranosa possa ser a possível causa principal da hipoplasia do lobo esquerdo porém questionam se ela realmente prejudica o desenvolvimento do lobo esquerdo ou apenas ocupa o espaço do lobo esquerdo hipoplásico.

Resumo dos casos publicados na literatura inglesa de 1985 a 2007 é mostrado na Tabela 1.

TABELA 1 - Casos relatados na literatura

\begin{tabular}{|c|c|c|c|c|c|}
\hline Autores & Ano & $\begin{array}{l}\text { I d a d e } \\
\text { Sexo }\end{array}$ & $\begin{array}{l}\text { Agenesia } \\
\text { Hipo }\end{array}$ & $\begin{array}{l}\text { Anomalias } \\
\text { associadas }\end{array}$ & Comentários \\
\hline Champetier $^{2}$ & 1985 & $55 / \mathrm{F}$ & $\mathrm{H}$ & $\begin{array}{l}\text { Hipoplasia da cúpula } \\
\text { diafragmática } \mathrm{E}\end{array}$ & \\
\hline Champetier $^{2}$ & 1985 & $25 / \mathrm{F}$ & $\mathrm{H}$ & Lobo acessório & \\
\hline Ahmed $^{1}$ & 1988 & $57 / \mathrm{M}$ & A & Volvo gástrico & Etilismo crônico \\
\hline Kakitsubata $^{8}$ & 1991 & $55 / \mathrm{M}$ & NR & - & Fadiga \\
\hline Kakitsubata $^{8}$ & 1991 & $60 / \mathrm{M}$ & NR & - & Tumor ureteral \\
\hline Kakitsubata $^{8}$ & 1991 & $63 / \mathrm{M}$ & NR & - & $\begin{array}{l}\text { Câncer pulmão, } \\
\text { pólipo colon }\end{array}$ \\
\hline Kakitsubata $^{8}$ & 1991 & $68 / \mathrm{F}$ & NR & $\begin{array}{l}\text { Aumento lobo } \\
\text { caudado }\end{array}$ & $\begin{array}{l}\text { He mang io ma } \\
\text { hepático }\end{array}$ \\
\hline Kakitsubata $^{8}$ & 1991 & $68 / \mathrm{F}$ & NR & - & Parkinson \\
\hline Ozgun $^{15}$ & 1992 & $69 / \mathrm{M}$ & NR & None & $\begin{array}{l}\text { Distúrbio esqui- } \\
\text { zoafetivo }\end{array}$ \\
\hline Maeda $^{11}$ & 1998 & $71 / \mathrm{F}$ & $\mathrm{H}$ & Vesícula flutuante & Hepatite C \\
\hline Zenda $^{18}$ & 2000 & $69 / \mathrm{M}$ & $\mathrm{H}$ & $\begin{array}{l}\text { Hérnia Bochdalek D/ } \\
\text { Vesicula flutuante }\end{array}$ & \\
\hline Ohkubo $^{13}$ & 2001 & $90 / \mathrm{F}$ & $\mathrm{H}$ & $\begin{array}{l}\text { Sublobulação do cau- } \\
\text { dado e quadrado }\end{array}$ & \\
\hline Saigusa ${ }^{17}$ & 2001 & $\begin{array}{l}78 / \mathrm{F} \\
\text { Autópsia }\end{array}$ & $\mathrm{H}$ & Ausência de vesícula & \\
\hline $\mathrm{Cho}^{3}$ & 2003 & $\begin{array}{l}\text { 59/F } \\
\text { Autópsia }\end{array}$ & $\mathrm{H}$ & $\begin{array}{l}\text { Ausência lobo } \\
\text { Spiegel }\end{array}$ & Câncer gastrico \\
\hline Lee $^{10}$ & 2004 & $\begin{array}{l}75 / \mathrm{F} \\
\text { Autópsia }\end{array}$ & $\mathrm{H}$ & - & Pneumonia \\
\hline Lee ${ }^{10}$ & 2004 & $\begin{array}{l}\text { 91/F } \\
\text { Autópsia }\end{array}$ & $\mathrm{H}$ & - & Infarto cerebral \\
\hline Este caso & 2004 & $48 / \mathrm{F}$ & A & - & Obesidade Mórbida \\
\hline
\end{tabular}

NR: não relatadas

\section{CONCLUSÃO}

Embora as alterações anatômicas hepáticas sejam raramente encontradas, elas já estão bem descritas na literatura. As alterações pouco interferem com a qualidade de vida do paciente e não devem influenciar as escolhas táticas do cirurgião. 
Martins BC, Ramos MFKP, Cleva R, Zilberstein B. Incidental finding of agenesis of the left hepatic lobe in a patient with acute cholecystitis. ABCD Arq Bras Cir Dig 2008;21(4): 208-10

ABSTRACT - Introduction - Anomalies of hepatic morphology are rare entities caused by abnormal embryological development of the liver. They can be classified as anomalies due to defective development (agenesis, aplasia or hypoplasia) or anomalies due to excessive development of the liver (accessory lobes). Case report - A 48 years old obese female looked for emergency assistance with acute cholecystitis. An incidental finding of left hepatic lobe agenesis was observed when the patient was submitted to laparoscopic cholecystectomy. There was no hepatic parenchyma to the left of falciform ligament and gallbladder was settled on its normal position. Cholecystectomy was performed uneventfully and an adjustable gastric band was placed for the treatment of the morbid obesity. The patient was discharged after 60 hours.

HEADINGS - Cholecystectomy. Digestive system abnormalities.

\section{REFERÊNCIAS}

1. Ahmed AF, Bediako AK, Rai D. Agenesis of the left hepatic lobe with gastric volvulus. N Y State J Med. 1988 Jun;88(6):327-8.

2. Champetier J, Yver R, Létoublon C, Vigneau B. A general review of anomalies of hepatic morphology and their clinical implications. Anat Clin. 1985;7(4):28599.

3. Cho YH, Murakami G, Lee MS, Song CH, Han EH, Jin ZW, Cho BH. Hypoplasia of the left portal vein territory of the human liver: a case study. J Korean Med Sci. 2003 Dec;18(6):828-32.

4. Csendes A, Burdiles P, Smok G, Csendes P, Burgos A, Recio M. Histologic findings of gallbladder mucosa in 87 patients with morbid obesity without gallstones compared to 87 control subjects. J Gastrointest Surg. 2003 MayJun;7(4):547-51.

5. Elias H, Petty D. Gross anatomy of the blood vessels and ducts within the human liver. Am J Anat. 1952 Jan;90(1):59-111.

6. Heller A. Mangelhafte Entwicklung des rechten Leperlappens. Virchows Arch. 1870;51:355-7.

7. Inoue T, Ito Y, Matsuzaki Y, Okauchi Y, Kondo H, Horiuchi N, Nakao K, Iwata M. Hypogenesis of right hepatic lobe accompanied by portal hypertension: case report and review of 31 Japanese cases. J Gastroenterol. 1997 Dec;32(6):836-42.

8. Kakitsubata Y, Nakamura R, Mitsuo H, Suzuki Y, Kakitsubata S, Watanabe $\mathrm{K}$. Absence of the left lobe of the liver: US and CT appearance. Gastrointest Radiol. 1991;16(4):323-5.
9. Kovarik JL, Jensen NK. Congenital aplasia of the right hepatic lobe with right-sided diaphragmatic hernia and intestinal malrotation. Int Surg. 1969 Jun;51(6):499-503.

10. Lee UY, Murakami G, Han SH. Left lobe hypoplasia of the human liver: a report of two cases. Surg Radiol Anat. 2004 Oct;26(5):417-23.

11. Maeda N, Horie Y, Shiota G, Suou T, Andachi H, Kawasaki H. Hypoplasia of the left hepatic lobe associated with floating gallbladder: a case report. Hepatogastroenterology. 1998 Jul-Aug;45(22):1100-3.

12. Marchal G, Bertrand L. Volvulus intermittent de le stomac et absence de lobe gauche du foie. J Chir. 1966;92:461-72.

13. Merril GC. Complete absence of the left lobe of the liver. Arch Pathol. 1946; 42:232-3.

14. Messing A, Ashley-Montagu MF. A note on a case of the left lobe of the liver in man. Anat Rec. 1932;53:169-72.

15. Ozgun B, Warshauer DM. Absent medial segment of the left hepatic lobe: CT appearance. J Comput Assist Tomogr. 1992 Jul-Aug;16(4):666-8.

16. Pages A, Marty-Double Ch. Les malformations constitutuionnelles du foie et des voies biliaires intrahépatiques. Ed Varia, Paris.1972;19-43.

17. Radin DR, Colletti PM, Ralls PW. Agenesis of the right lobe of the liver. Radiology. 1987; 164:639-42.

18. Rolleston HD. Specimens of livers with anomalies in their lobulation. J Anat Physiol. 1893;27:577-81.
Fonte de financiamento: não há Conflito de interesse: não há Recebido para publicação: 15/06/2008 Aceito para publicação: 30/08/2008 